\title{
A COUPLED LOCALIZED RBF MESHLESS/DRBEM FORMULATION FOR ACCURATE MODELING OF INCOMPRESSIBLE FLUID FLOWS
}

\author{
LEONARDO A. BUENO ${ }^{1}$, EDUARDO A. DIVO $^{1}$ \& ALAIN J. KASSAB ${ }^{2}$ \\ ${ }^{1}$ Department of Mechanical Engineering, Embry-Riddle Aeronautical University, Daytona Beach, FL, USA. \\ ${ }^{2}$ Department of Mechanical and Aerospace Engineering University of Central Florida, Orlando, FL, USA.
}

\begin{abstract}
Velocity-pressure coupling schemes for the solution of incompressible fluid flow problems in Computational Fluid Dynamics (CFD) rely on the formulation of Poisson-like equations through projection methods. The solution of these Poisson-like equations represent the pressure correction and the velocity correction to ensure proper satisfaction of the conservation of mass equation at each step of a time-marching scheme or at each level of an iteration process. Inaccurate solutions of these Poisson-like equations result in meaningless instantaneous or intermediate approximations that do not represent the proper time-accurate behavior of the flow. The fact that these equations must be solved to convergence at every step of the overall solution process introduces a major bottleneck for the efficiency of the method. We present a formulation that achieves high levels of accuracy and efficiency by properly solving the Poisson equations at each step of the solution process by formulating a Localized RBF Collocation Meshless Method (LRC-MM) solution approach for the approximation of the diffusive and convective derivatives while employing the same framework to implement a Dual-Reciprocity Boundary Element Method (DR-BEM) for the solution of the ensuing Poisson equations. The same boundary discretization and point distribution employed in the LRC-MM is used for the DR-BEM. The methodology is implemented and tested in the solution of a backwardfacing step problem.
\end{abstract}

Keywords: dual reciprocity boundary element method, incompressible fluid flows, meshless methods, radial basis functions.

\section{INTRODUCTION}

Meshless methods are numerical techniques that utilize interpolation, either global or localized, on a set of non-ordered points, see $[1,2]$. These are being used to resolve complex problems, such as flow transport problems, see [3]. There are several advantages to them such as accuracy control, since additional nodes made be added where needed, and a more accurate representation of the objects through meshfree discretization, see [4]. The principal reason for such flexibility is that the nodes do not need to conformant to the geometry and since elements are not used to join the nodes, degeneration is a non-issue. This is a direct contrast to meshed techniques, which required that node connectivity follows the boundary contours and thus there is a need to have well-shaped elements, see [5]. Truly meshless methods do not require any mesh or particular node distribution. Global radial-basis function (RBF) interpolation meshless methods are computationally expensive and require special treatment to deal with poor conditioning in the algebraic set of equations. The localized RBF collocation meshless methods (LRC-MM) address the issues that arise from the global RBF approach, see [1, 6]. LRC-MM have been implemented in the solution of incompressible fluid flows effectively adapting upwinding schemes for convective-dominated flows, see $[1,3,5,6]$. However, in decoupling the governing equations for incompressible fluid flows, a Poisson-like equation arises for the solution of the pressure field at each time step. Although 
LRC-MM have proven to be an accurate solution approach for Poisson equations, issues are still present with their efficiency and their ability to accurately capture the derivative fields, which are crucial to the proper updating of the velocity field for a stable incompressible fluid flow solution scheme.

To mitigate the issues arising from the solution of a Poisson equation at each step of the time-progressing process of incompressible fluid flows, a Dual Reciprocity Boundary Element Method (DR-BEM) is proposed. In the DR-BEM, the domain integral remaining from the generation term of the Poisson equation is transferred to the boundary via an RBF expansion, see [7-9]. This results in a Boundary-only integral equation which can be numerically implemented in the same point distribution being employed for the LRC-MM. Furthermore, the resulting algebraic set of the DR-BEM can be decomposed to be used throughout the time-stepping process in an efficient manner as the boundary discretization and internal point distribution do not change. Moreover, DR-BEM have been shown to produce a very accurate solution and derivative fields, see [7-9], which is of premium importance in updating the velocity field at every step of the time-stepping process of the incompressible fluid flow solution scheme. DR-BEM have been effectively applied to solve diffusion-like and non-linear heat conduction problems through the same approach, see [7-9].

\section{GOVERNING EQUATIONS FOR INCOMPRESSIBLE FLUID FLOWS}

The set of equations that define incompressible fluid flows correspond to mass, momentum and energy conservation in the form of the Navier-Stokes equations as shown below:

$$
\begin{aligned}
& \nabla \cdot \vec{V}=0 \\
& \rho \frac{\partial \vec{V}}{\partial t}+\rho(\vec{V} \cdot \nabla) \vec{V}=\mu \nabla^{2} \vec{V}-\nabla p+\vec{f} \\
& \rho c_{p} \frac{\partial T}{\partial t}+\rho c_{p}(\vec{V} \cdot \nabla) T=k \nabla^{2} T+\dot{u}_{g}^{\prime \prime}
\end{aligned}
$$

Where $\rho$ is the density $\left[\mathrm{Kg} / \mathrm{m}^{3}\right], \mu$ is the dynamic viscosity $\left[\mathrm{N} \cdot \mathrm{s} / \mathrm{m}^{2}\right], \vec{f}$ is the body force $\left[\mathrm{N} / \mathrm{m}^{3}\right], c_{p}$ is the specific heat $[\mathrm{kJ} / \mathrm{kg} \mathrm{K}], k$ is the thermal conductivity $[\mathrm{W} / \mathrm{mK}]$, and $\dot{u}_{g}^{\prime \prime}$ is the energy generation $\left[\mathrm{W} / \mathrm{m}^{3}\right]$. The space and time- dependent field variables are the components of the velocity vector $\vec{V}(x, t)[\mathrm{m} / \mathrm{s}]$, the pressure $p(x, t)$, and the temperature $T(x, t)$. Note that there is a lack of an explicit equation for the pressure $p(x, t)$ which must now be indirectly constrained to advance the solution in time. The solution can be discretized in time such that $t_{n}=n \Delta t$, and position the different terms at different time steps as shown below (for the mass and momentum equations):

$$
\begin{aligned}
& \nabla \cdot \vec{V}^{n+1}(x)=0 \\
& \rho \frac{\partial \vec{V}^{n}(x)}{\partial t}+\rho\left(\vec{V}^{n}(x) \cdot \nabla \vec{V}^{n}(x)\right)=\mu \nabla^{2} \vec{V}^{n}(x) \\
& -\nabla p^{n+1}(x)+\vec{f}^{n}(x)
\end{aligned}
$$

Expressing the pressure at time step $t_{n+1}$ in terms of the pressure at time step $t_{n}$ and a pressure correction field $\phi^{n}(x)$ as:

$$
p^{n+1}(x)=p^{n}(x)+\phi^{n}(x)
$$


Approximating the time derivative in the momentum equation in eqn (2) with a backward differencing scheme, introducing eqn (3) into eqn (2), and rearranging results in:

$$
\begin{array}{r}
\begin{array}{r}
\nabla \cdot \vec{V}^{n+1}(x)=0 \\
\vec{V}^{n+1}(x)+\frac{\Delta t}{\rho} \nabla \phi^{n}(x)=\vec{V}^{n}(x) \\
+\Delta t\left[\frac{\mu}{\rho} \nabla^{2} \vec{V}^{n}(x)-\frac{1}{\rho} \nabla p^{n}(x)\right. \\
\left.+\frac{1}{\rho} \vec{f}^{n}(x)-\left(\vec{V}^{n}(x) \cdot \nabla \vec{V}^{n}(x)\right)\right]
\end{array}
\end{array}
$$

An intermediate velocity field $\vec{V}^{*}(x)$ can be defined as:

$$
\vec{V}^{*}(x)=\vec{V}^{n+1}(x)+\frac{\Delta t}{\rho} \nabla \phi^{n}(x)
$$

Leading to the discretized momentum equation for the intermediate velocity field $\vec{V}^{*}(x)$ as:

$$
\begin{array}{r}
\vec{V}^{*}(x)=\vec{V}^{n}(x)+\Delta t\left[\frac{\mu}{\rho} \nabla^{2} \vec{V}^{n}(x)-\frac{1}{\rho} \nabla p^{n}(x)\right. \\
\left.+\frac{1}{\rho} \vec{f}^{n}(x)-\left(\vec{V}^{n}(x) \cdot \nabla \vec{V}^{n}(x)\right)\right]
\end{array}
$$

Introducing the intermediate velocity field $\vec{V}^{*}(x)$ in eqn (5) to the conservation of mass in eqn (4) leads to:

$$
\nabla \cdot\left(\vec{V}^{*}(x)-\frac{\Delta t}{\rho} \nabla \phi^{n}(x)\right)=0
$$

Resulting in a Poisson equation for the pressure correction field $\phi^{n}(x)$ as:

$$
\nabla^{2} \phi^{n}(x)=\frac{\rho}{\Delta t}\left(\nabla \cdot \vec{V}^{*}(x)\right)
$$

Equation (8) must be solved at every time-step $t_{n}$ so that the pressure field $p^{n}(x)$ is corrected to $p^{n+1}(x)$ through eqn (3) and the intermediate velocity field $\vec{V}^{*}(x)$ is corrected to $\vec{V}^{n+1}(x)$ through eqn (5).

This particular step imposes a stability concern in the solution process as an accurate approximation of the pressure correction field $\phi^{n}(x)$ and its derivatives is imperative to properly advance the pressure and velocity fields to the new time step $t_{n+1}$. In order to mitigate this concern, an accurate Dual-Reciprocity Boundary Element Method (DR-BEM) will be implemented to solve the Poisson equation in (8) for the pressure correction field $\phi^{n}(x)$ while coupling it with a Localized RBF Collocation Meshless Method (LRC-MM) to approximate the space operators in the explicit form of the discretized momentum equation in (6). The two solution methods will be implemented in the same collocation point distribution eliminating the need to interpolate data. 


\section{LOCALIZED RBF COLLOCATION MESHLESS METHOD (LRC-MM)}

Evaluation of the field variable derivatives in the discretized momentum equation in (6) (i.e. $\nabla p(x), \vec{V}(x) \cdot \nabla \vec{V}(x)$, and $\left.\nabla^{2} \vec{V}(x)\right)$ will be accomplished by formulating an accurate and efficient meshless method. Radial-Basis Function (RBF) collocation meshless methods use a scattered point distribution on the boundary and in the domain. RBF interpolation is used to expand the field variable(s) over the node distribution so there is no need for meshing or structuring of the point distribution [1]. The expansion of a field variable, $p(x)$, in terms of RBFs, $\chi(x)$, is given by:

$$
p(x)=\sum_{j=1}^{N} \alpha_{j} \chi_{j}(x)
$$

Where, $\chi_{j}(x)$ is the prescribed $j^{t h} \mathrm{RBF}$ expansion function and $\alpha_{j}$ represent the expansion coefficients, found through the standard collocation of the field variable. For instance, the inverse Multiquadrics RBFs can be used as the expansion functions having the form:

$$
\chi_{j}(x)=\left[\left(\frac{r_{j}(x)}{c}\right)^{2}+1\right]^{-\frac{1}{2}}
$$

Here, $r_{j}(x)$ is the Euclidian distance from the $j^{\text {th }}$ collocation point, $x_{j}$, to any field point, $x$, while $c$ is a shape parameter used to control the flatness or steepness of the RBF.

Crucially, $N$ is the total number of collocation points in the domain and on the boundary, which, in the case of global expansion of the field variable, results in a system of equations of the form:

$$
\{p\}=[C]\{\alpha\}
$$

As such, the size of the resulting collocation matrix $[C]$ is $N$. Since the coefficients, $\alpha_{j}$, are found by solving eqn (11), it becomes more computationally intensive to solve as the number of total collocation nodes increases with the complexity of the model [1]. Another issue that contributes to the inefficiency of the method is the need for optimization of the shape parameter $c$ in eqn (10). [2, 3]

This is where the Localized RBF Collocation Meshless Method (LRC-MM) can be helpful. This effective approach relies on an expansion of the field variable over a local area of influence or localized topology, $\Omega_{f}$, consisting of a number $N_{f}$ of influence collocation points around the data center $x_{c}$. The localized expansion of the field variable $p(x)$ using RBFs can then be expressed as:

$$
p(x)=\sum_{j=1}^{N_{f}} \alpha_{j} \chi_{j}(x)
$$

Collocation of this expansion on the $N_{f}$ influence points of the topology results in a system of equations of the form:

$$
\{p\}_{N_{f}, 1}=[C]_{N_{f}, N_{f}}\{\alpha\}_{N_{f}, 1}
$$

It is important to note that the collocation matrix $[C]$ is of size $N_{f}$ and therefore the expansion coefficients $\{a\}$ can be solved for more efficiently as:

$$
\{\alpha\}=[C]^{-1}\{p\}
$$


This would mean a significant reduction in the computational workload when compared to a solution that involves a collocation matrix of size $N$. In addition, any differential operator $L$ applied to the field variable $p(x)$ at the topology data center $x_{c}$ can be expressed with the expansion in eqn (12) as:

$$
L p\left(x_{c}\right)=\sum_{j=1}^{N_{f}} \alpha_{j} L \chi_{j}\left(x_{c}\right)
$$

Or in vector-vector form as:

$$
L p_{c}=\left\{L \chi_{c}\right\}^{T}\{\alpha\}
$$

Introducing the expansion coefficient expression in eqn (14) results in:

$$
L p_{c}=\left\{L \chi_{c}\right\}^{T}[C]^{-1}\{p\}
$$

Defining the interpolation vector $\left\{L_{c}\right\}$ as:

$$
\left\{L_{c}\right\}^{T}=\left\{L \chi_{c}\right\}^{T}[C]^{-1}
$$

Which can be pre-built and stored prior to the solution process.

This formulation leads to the simple form for the evaluation of any differential operator $L$ applied to the field variable $p(x)$ at the topology data center $x_{c}$ :

$$
L p_{c}=\left\{L_{c}\right\}^{T}\{p\}
$$

Notice that the expression in eqn (19) is a simple vector-vector inner product that can be executed very efficiently at each collocation point to evaluate the derivatives of any field variable, such as those necessary to approximate the solution of the explicit momentum equation in (6). I.e. $\nabla p(x), \vec{V}(x) \cdot \nabla \vec{V}(x)$, and $\nabla^{2} \vec{V}(x)$.

\section{DUAL-RECIPROCITY BOUNDARY ELEMENT METHOD (DR-BEM)}

The solution of the Poisson equation in (8) for the pressure correction $\phi^{n}(x)$ required at every time-step of the solution process will be accomplished by an accurate Dual-Reciprocity Boundary Element Method (DR-BEM) over the same point distribution employed for the LRC-MM.

Recast the Poisson equation in (8) as:

$$
\nabla^{2} \phi^{n}(x)=-B(x) \quad \therefore \quad B(x)=\frac{-\rho}{\Delta t} \nabla \cdot \vec{V}^{*}(x)
$$

Multiplying eqn (20) by a weight function $G(x, \zeta)$ and integrating over the problem domain $\Omega$ results in:

$$
\int_{\Omega} \nabla^{2} \phi^{n}(x) G(x, \zeta) d \Omega=-\int_{\Omega} B(x) G(x, \zeta) d \Omega
$$

Applying Green's second identity on the left-hand side leads to:

$$
\begin{aligned}
& \int_{\Omega} \phi^{n}(x) \nabla^{2} G(x, \zeta) d \Omega-\oint_{\Gamma} \phi^{n}(x) \frac{\partial G(x, \zeta)}{\partial n} d \Gamma \\
& \quad+\oint_{\Gamma} G(x, \zeta) \frac{\partial \phi^{n}(x)}{\partial n} d \Gamma=-\int_{\Omega} B(x) G(x, \zeta) d \Omega
\end{aligned}
$$


Now let:

$$
\nabla^{2} G(x, \zeta)=-\delta(x, \zeta)
$$

Where $\delta(x, \zeta)$ is the Dirac delta function with field point $x$ and source point $\zeta$. Equation (23) can be solved analytically in any dimension for the Green's Free-space solution. For instance, in 2D:

$$
G(x, \zeta)=-\frac{1}{2 \pi} \ln |x-\zeta|
$$

This leads to the integral equation for the field variable $\phi^{n}(\zeta)$ at any point $\zeta$ within the domain and boundary as:

$$
c(\zeta) \phi^{n}(\zeta)+\oint_{\Gamma} \phi^{n}(\zeta) H(x, \zeta) d \Gamma=\oint_{\Gamma} q^{n}(x) G(x, \zeta) d \Gamma+b(\zeta)
$$

Where:

$$
\begin{aligned}
& q^{n}(x)=\frac{\partial \phi^{n}(x)}{\partial n} \\
& H(x, \zeta)=\frac{\partial G(x, \zeta)}{\partial n} \\
& b(\zeta)=\int_{\Omega} B(x) G(x, \zeta) d \Omega \\
& c(\zeta)=\int_{\Omega} \delta(x, \zeta) d \Omega
\end{aligned}
$$

The parameter $c(\zeta)$ is equal to 1 in the interior and is equal to 0.5 on smooth boundaries.

A discretization of the boundary $\Gamma$ is now introduced to numerically approximate the boundary integrals in eqn (25). For instance, let the boundary $\Gamma$ be subdivided into $N_{B}$ constant boundary elements such that:

$$
\Gamma=\sum_{j=1}^{N_{B}} \Delta \Gamma_{j}
$$

Then, introducing this discretization in the boundary integral eqn (25) results in the boundary integral equation (BIE):

$$
c(\zeta) \phi^{n}(\zeta)+\sum_{j=1}^{N_{B}} \phi_{j}^{n} \int_{\Delta \Gamma_{j}} H(x, \zeta) d \Gamma=\sum_{j=1}^{N_{B}} q_{j}^{n} \int_{\Delta \Gamma_{j}} G(x, \zeta) d \Gamma+b(\zeta)
$$

Collocating the source point $\zeta$ at $i=1 \ldots N_{B}$ points on the boundary $\Gamma$ leads to:

Where:

$$
c_{i} \phi_{i}^{n}+\sum_{j=1}^{N_{B}} \hat{H}_{i j} \phi_{j}^{n}=\sum_{j=1}^{N_{B}} G_{i j} q_{j}^{n}+b_{i}
$$

$$
\begin{aligned}
& \hat{H}_{i j}=\int_{\Delta \Gamma_{j}} H\left(x, \zeta_{i}\right) d \Gamma \\
& G_{i j}=\int_{\Delta \Gamma_{j}} G\left(x, \zeta_{i}\right) d \Gamma \\
& b_{i}=b\left(\zeta_{i}\right)=\int_{\Omega} B(x) G\left(x, \zeta_{i}\right) d \Omega
\end{aligned}
$$


It is noted that the point distribution for the DR-BEM need not be changed from that of the LRC-MM, therefore the boundary elements $\Delta \Gamma_{j}$ and collocation points $\zeta_{i}$ in eqn (30) coincide with the boundary discretization established for the LRC-MM. The discretized BIE in eqn (29) is rearranged in matrix-vector form as:

$$
[H]_{N_{B}, N_{B}}\left\{\phi^{n}\right\}_{N_{B}, 1}=[G]_{N_{B}, N_{B}}\left\{q^{n}\right\}_{N_{B}, 1}+\{b\}_{N_{B}, 1}
$$

Introducing boundary conditions for $\phi^{n}$ the expression above can be rearranged into a linear system of equations $[A]\{x\}=\{d\}$ where the elements of the vector $\{x\}$ are the unknown values of $\phi^{n}$ or $q^{n}$ on the boundary.

The elements of the vector $b_{i}=\{b\}$ depend on the right-hand side of the Poisson equation $B(x)$. As $B(x)$ is explicitly known but non-homogeneous throughout the domain, special care must be taken when evaluating the domain integral for $b_{i}$ in eqn (30). Let the term $B(x)$ be expanded in terms of expansion functions $f_{k}(x)$ as:

$$
B(x)=\sum_{k=1}^{N} \alpha_{k} f_{k}(x)
$$

Where $N$ is the total number of points on the boundary and in the interior of the domain, $N=N_{B}+N_{I}$. This point distribution corresponds exactly to the one employed for the LRC-MM and the boundary points $N_{B}$ correspond with the boundary element discretization.

Now let the expansion functions be such that:

$$
f_{k}(x)=\nabla^{2} u_{k}(x)
$$

As in the LRC-MM, RBFs will be employed as the expansion functions of choice, however, the inverse Multiquadrics RBFs in eqn (10) cannot be employed directly because an analytical solution for $u_{k}(x)$ from (33) is not available. Instead, the Polyharmonic RBF will be used as:

$$
f_{k}(x)=\frac{r_{k}(x)}{c}+1
$$

For which the corresponding function $u_{k}(x)$ can readily be found. Introducing eqn (32) and eqn (33) in eqn (30), the elements of vector $b_{i}$ become:

$$
b_{i}=\sum_{k=1}^{N} \alpha_{k} \int_{\Omega} \nabla^{2} u_{k}(x) G\left(x, \zeta_{i}\right) d \Omega
$$

Applying the Green's 2nd identity to the right-hand side of eqn (35) results in a transformation to boundary integrals only as:

$$
b_{i}=\sum_{k=1}^{N} \alpha_{k}\left[\oint_{\Gamma} p_{k}(x) G\left(x, \zeta_{i}\right) d \Gamma-\oint_{\Gamma} u_{k}(x) H\left(x, \zeta_{i}\right) d \Gamma-c\left(\zeta_{i}\right) u_{k}\left(\zeta_{i}\right)\right]
$$

Where $p_{k}(x)=\partial u_{k}(x) / \partial n$. The same boundary discretization into $N_{B}$ constant boundary elements is introduced now to approximate the boundary integrals above resulting in:

$$
b_{i}=\sum_{k=1}^{N} \alpha_{k}\left[\sum_{j=1}^{N_{B}} G_{i j} p_{j}^{k}-\sum_{j=1}^{N_{B}} H_{i j} u_{j}^{k}\right]
$$


The expansion coefficients $\alpha_{k}$ can be determined by collocating the expansion in eqn (32) at $x=x_{l} \because l=1 \ldots N$ so that:

$$
\{B\}_{N, 1}=[F]_{N, N}\{\alpha\}_{N, 1}
$$

Once the boundary value problem for $\phi^{n}$ is solved, the discretized BIE in (28) can be employed to evaluate the value of $\phi^{n}(\zeta)$ at any interior point $\zeta$. Furthermore, the derivatives of $\phi^{n}$ at any interior point $\zeta$ can required for updating the velocity field in eqn (5) can be accurately evaluated by differentiating the BIE in (28), for instance:

$$
\frac{\partial \phi^{n}(\zeta)}{\partial \zeta_{x}}+\sum_{j=1}^{N_{B}} \phi_{j}^{n} \int_{\Delta \Gamma_{j}} \frac{\partial H(x, \zeta)}{\partial \zeta_{x}} d \Gamma=\sum_{j=1}^{N_{B}} q_{j}^{n} \int_{\Delta \Gamma_{j}} \frac{\partial G(x, \zeta)}{\partial \zeta_{x}} d \Gamma+\frac{\partial b(\zeta)}{\partial \zeta_{x}}
$$

\section{NUMERICAL EXAMPLE}

To test the LRC-MM/DR-BEM scheme, a numerical example is configured as a two-dimensional incompressible fluid flow problem in a backward-facing step as shown below, where the geometrical and thermophysical parameters are: length $L=1 \mathrm{~m}$, height $H=0.1 \mathrm{~m}$, step height $h=0.04 \mathrm{~m}$, the fluid is air with density $\rho=1.205 \frac{\mathrm{kg}}{\mathrm{m}^{3}}$, viscosity $\mu=2 \cdot 10^{-5} \frac{\mathrm{kg}}{\mathrm{m} \cdot \mathrm{s}}$, thermal conductivity $k=0.0257 \frac{\mathrm{W}}{\mathrm{mK}}$, and specific heat $c_{p}=1005 \frac{\mathrm{J}}{\mathrm{kgK}}$.

Three simulations will be executed at three different values of the Reynolds number based on the step size $\left(\operatorname{Re}_{h}=\rho V h / \mu\right)$ such that: $\operatorname{Re}_{h}=10,100,1000$. A third-order upwinding scheme is implemented for the convective derivative on all topologies along the direction where the local Reynolds number $\left(\operatorname{Re}_{\Delta x}=\rho u \Delta x / \mu, \operatorname{Re}_{\Delta y}=\rho u \Delta y / \mu\right)$ exceeds 1 .

Constant boundary elements equally-spaced at $1 \mathrm{~cm}$ are distributed throughout the boundary with a layer of shadow internal points $0.5 \mathrm{~cm}$ away from each boundary node and an internal point distribution with uniform spacing at $1 \mathrm{~cm}$ are used for the LRC-MM and DR-BEM solutions as shown below:

The localized influence topologies are setup as a circle of radius $r_{f}=3.1 \mathrm{~cm}$ (slightly over 3 times the average spacing) around each data center to guarantee that at a minimum at least 3 points in each direction are included. A close-up of a topology (on an irregular point distribution) using this criteria is shown below:

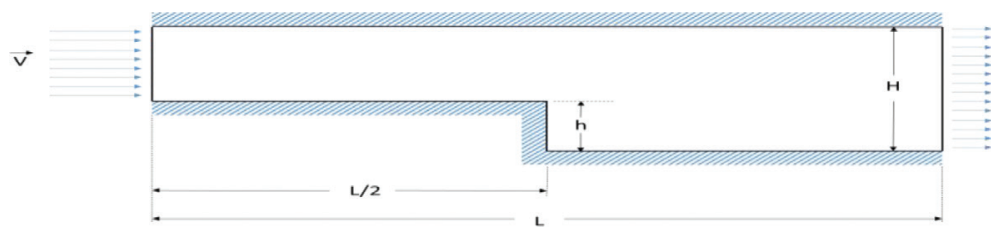

Figure 1: Geometric configuration of Backward-Facing step problem.

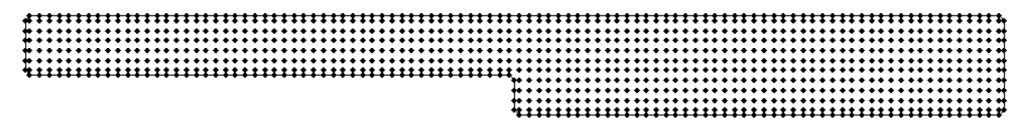

Figure 2: Meshless point distribution and boundary element discretization. 


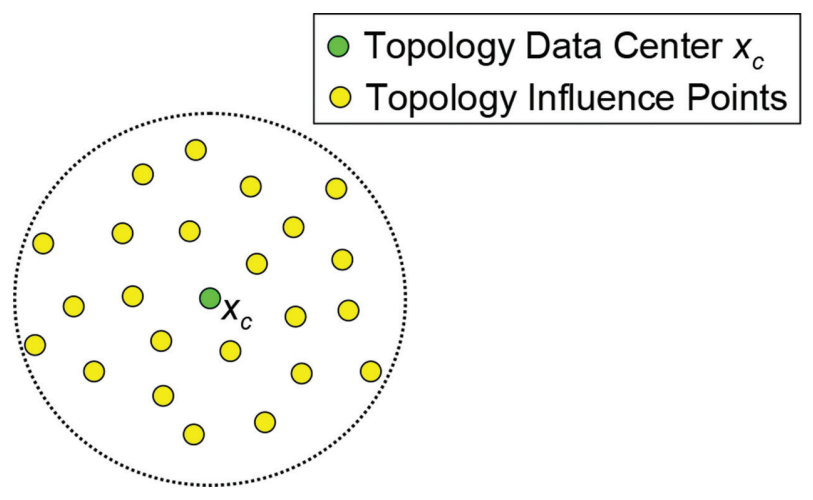

Figure 3: Typical localized topology of influence.
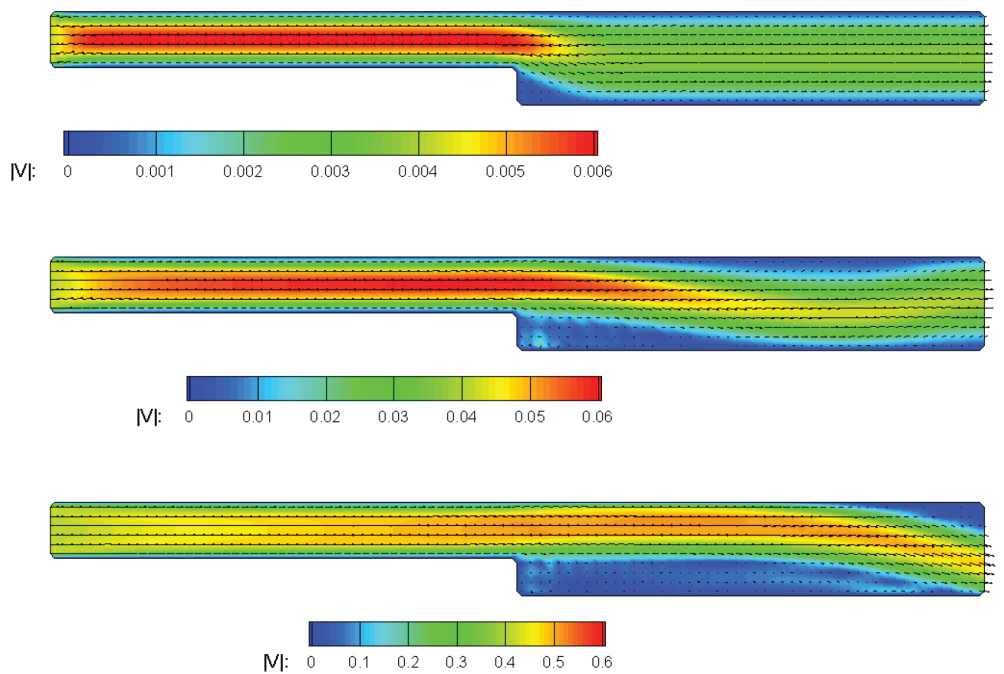

Figure 4: Velocity contours and vectors for $\operatorname{Re}=10, \operatorname{Re}=100$, and $\operatorname{Re}=1000$ after 200 s of real-time.

The velocity contours and vectors corresponding to the solution approximation after 200s of real-time are presented in the figure below for $\operatorname{Re}_{h}=10, \operatorname{Re}_{h}=100$, and $\operatorname{Re}_{h}=1000$. The reattachment points and recirculation zones can be identified from the figure showing stable numerical results.

\section{CONCLUSIONS}

A formulation is presented to implement a Dual-Reciprocity Boundary Element Method (DR-BEM) to accurately solve the pressure-correction Poisson equation that arises at each step of the solution process of incompressible fluid flows while the convective and diffusive derivatives of the Navier-Stokes equations are approximated by implementing a Localized RBF Collocation Meshless Method (LRC-MM). Inverse Multiquadrics RBF are employed for locally expanding the field in the LRC-MM while Polyharmonics RBF are employed for expanding the forcing term of the Poisson equation in the DR-BEM. The same boundary 
discretization and point distribution employed in the LRC-MM is used for the DR-BEM. The methodology is implemented and tested in the solution of a backward-facing step problem showing accurate and stable results in an efficient and robust framework that can be applied to a wide range of incompressible fluid flow problems.

\section{REFERENCES}

[1] Pepper, D.K., Kassab, A.J. \& Divo, E., An Introduction to Finite Element, Boundary Element, and Meshless Methods with Applications to Heat Transfer and Fluid Flow, ASME Press, 2014.

http://dx.doi.org/10.1115/1.860335

[2] Idelsohn, S. \& Oñate, E., To mesh or not to mesh. that is the question... Computer Methods in Applied Mechanics and Engineering, 195(37-40), pp. 4681-4696, 2006. http://dx.doi.org/10.1016/j.cma.2005.11.006

[3] Divo, E. \& Kassab, A.J., An efficient localized radial basis function Meshless method for fluid flow and conjugate heat transfer. Journal of Heat Transfer, 129(2), pp. 124136, 2007.

http://dx.doi.org/10.1115/1.2402181

[4] Li, S. \& Liu, W.K., Meshfree and particle methods and their applications. Applied Mechanics Review, 55(1), pp. 1-34, 2002.

http://dx.doi.org/10.1115/1.1431547

[5] Kelly, J.M., Divo, E.A. \& Kassab, A.J., A GPU-accelerated meshless method for twophase incompressible fluid flows. Engineering Analysis with Boundary Elements, 40, pp. 36-49, 2014.

http://dx.doi.org/10.1016/j.enganabound.2013.11.015

[6] Divo, E. \& Kassab, A.J., Localized meshless modeling of natural convective flows. Numerical Heat Transfer, Part B: Fundamentals, 53, pp. 487-509, 2008. http://dx.doi.org/10.1080/10407790802083190

[7] Partridge, P.W., Brebbia, C.A. \& Wrobel, L.C., The Dual Reciprocity Boundary Element Method, Boston: Computational Mechanics, 1992.

[8] Divo, E. \& Kassab, A.J, Transient Non-linear Heat Conduction Solution by a Dual Reciprocity Boundary. ASME International Mechanical Engineering Congress and Exposition, Heat Transfer, 1, 2014.

[9] Wrobel, L.C., Brebbia, C.A., The dual reciprocity boundary element formulation for nonlinear diffusion problems. Computer Methods in Applied Mechanics and Engineering, 65(2), pp. 147-164, 1987.

http://dx.doi.org/10.1016/0045-7825(87)90010-7 